\title{
Anomalous Behavior of Ru for Catalytic Oxidation: A Theoretical Study of the Catalytic Reaction $\mathrm{CO}+\frac{1}{2} \mathrm{O}_{2} \rightarrow \mathrm{CO}_{2}$
}

\author{
C. Stampfl and M. Scheffler \\ Fritz-Haber-Institut der Max-Planck-Gesellschaft, Faradayweg 4-6, D-14195 Berlin-Dahlem, Germany
}

(Received 15 April 1996)

\begin{abstract}
Recent experiments revealed an anomalous dependence of carbon monoxide oxidation at $\mathrm{Ru}(0001)$ on oxygen pressure and a particularly high reaction rate. Below we report density functional theory calculations of the energetics and reaction pathways of the speculated mechanism. We will show that the exceptionally high rate is actuated by a weakly but nevertheless well bound $(1 \times 1)$-oxygen adsorbate layer. Furthermore, it is found that reactions via scattering of gas-phase $\mathrm{CO}$ at the oxygen covered surface may play an important role. Our analysis reveals, however, that reactions via adsorbed CO molecules (the so-called Langmuir-Hinshelwood mechanism) dominate. [S0031-9007(97)02370-3]

PACS numbers: 68.35.-p, 82.65.Jv, 82.65.My
\end{abstract}

The oxidation of carbon monoxide at transition metal surfaces is one of the most extensively studied heterogeneous catalytic reactions (see, for example, [1-3], and references therein). Numerous investigations performed under ultra high vacuum (UHV) conditions for many different metal surfaces have shown that the reaction proceeds via the so-called Langmuir-Hinshelwood (L-H) mechanism, which means that the reaction takes place between chemisorbed reagents. Typical conditions for working catalysts, however, are higher pressure and temperature. Although it has been demonstrated that for a number of systems extrapolation of data over a wide pressure range is valid [4], such a conclusion cannot be generalized. Recent high gas pressure studies (e.g., at about 10 torr), for oxidizing conditions (i.e., at $\mathrm{CO} / \mathrm{O}_{2}$ pressure ratios <1) $[3,5,6]$ reported that $\mathrm{CO}_{2}$ production over $\mathrm{Ru}(0001)$ is anomalous: (1) The rate of $\mathrm{CO}_{2}$ production was found to be exceptionally high - significantly higher than at any other transition metal surface. Interestingly, under UHV $\mathrm{Ru}(0001)$ is by far the poorest catalyst [1]. (2) The measured kinetic data (activation energy and pressure dependencies) were found to be markedly different to those for other substrates, and in contrast to the other transition metal catalysts, Pt, Pd, Ir, and Rh, highest rates occurred for high concentrations of oxygen at the surface. (3) Almost no chemisorbed $\mathrm{CO}$ was detected during or after the reaction. It was therefore speculated that the Eley-Rideal (E-R) mechanism is operational as opposed to the "usual" L-H mechanism [6]. In the E-R mechanism, the reaction occurs between gas-phase and chemisorbed particles. So far E-R mechanisms have been observed only experimentally for somewhat artificial reactions triggered by a beam of atomic hydrogen (or deuterium) [7]. To gain understanding into the drastically different behavior of $\mathrm{Ru}(0001)$ for the $\mathrm{CO}$ oxidation reaction, we carried out density functional theory (DFT) calculations, where the main approximations are the supercell approach and the employed functional for the exchange-correlation interaction. For the latter we use the generalized gradient approximation (GGA) [8] which is the best justified treatment to date. Our study represents the first theoretical attempt to follow a heterogenous catalytic reaction [molecular and dissociative (atomic) adsorption, surface reaction, desorption of products] using DFT-GGA and an extended surface. In brief, the details of the theoretical approach are summarized as follows: We use ab initio, fully separable, norm-conserving DFT-GGA pseudopotentials [9]. The GGA is thus treated in a consistent way, from the free atom to the solid surface and the reactants. Relativistic effects are taken into account by using spin averaged potentials. The surface calculations are performed using a $(2 \times 2)$ surface unit cell, a four atomic layer $\mathrm{Ru}$ slab, and a vacuum region corresponding to thirteen such layers. We use an energy cutoff of $40 \mathrm{Ry}$ with three special $\mathbf{k}$ points [10] in the two-dimensional Brillouin zone. Convergence tests for $\mathrm{O}$ on $\mathrm{Ru}(0001)$ indicated that this basis set provides a sufficiently accurate description [11]. The adsorbate structures are created on one side of the slab [12] where we relax the position of all the atoms using a damped molecular dynamics [13], except for the Ru atoms in the bottom two layers, which are kept at their bulklike positions. Details of the calculations will be published elsewhere [14].

It is well know that under UHV conditions, at room temperature, dissociative adsorption of $\mathrm{O}_{2}$ results in a saturation coverage of $\Theta_{\mathrm{O}} \approx 1 / 2$ corresponding to the formation of a $(2 \times 1)$ structure [15]. Recently, from DFT-GGA calculations we predicted that an even higher coverage should be stable on the surface, namely, a $(1 \times$ 1) structure with coverage $\Theta_{O}=1$, where the $O$ atoms occupy hcp-hollow sites [11]. Subsequently, this structure was indeed successfully created under UHV conditions [16], where it was concluded that formation of the $\Theta_{\mathrm{O}}=$ 1 structure from gas-phase $\mathrm{O}_{2}$ is hindered kinetically $[11,16]$, but by offering atomic oxygen (or under high pressure conditions), this phase can be attained. We also carried out calculations involving higher oxygen coverages on the surface, as well as geometries involving 
subsurface oxygen; these structures were found to be unstable and metastable, respectively, with respect to gasphase $\mathrm{O}_{2}$. We therefore do not expect them to play an important role for the present investigation. We refer to Refs. [16,17] for more details. Because the conditions under which the particularly high rates of $\mathrm{CO}_{2}$ formation occur involve elevated partial gas pressures (and $\mathrm{CO} / \mathrm{O}_{2}$ ratios $<1$ ), there will be a significant attempt frequency to overcome activation barriers for dissociative adsorption of $\mathrm{O}_{2}$. Thus it is likely that during reaction the oxygen coverage on the surface approaches one monolayer. We therefore initially assume in our investigation of the oxidation of $\mathrm{CO}$ at $\mathrm{Ru}(0001)$ that the $(1 \times 1)$ phase covers the surface.

As mentioned above, from the experiments it had been speculated that CO may react from the gas phase with adsorbed oxygen (the E-R mechanism) [6]. To investigate this possibility we first ask whether $\mathrm{CO}$ can adsorb on the $(1 \times 1)-\mathrm{O} / \mathrm{Ru}(0001)$ surface. The sites considered were the on-top and fcc-hollow sites, with respect to the $\mathrm{Ru}(0001)$ substrate, and a bridge site between two adsorbed $\mathrm{O}$ atoms (compare inset of Fig. 1). For each site we calculated the energy as a function of distance of the molecule from the surface. In these calculations the $\mathrm{CO}$ axis is held perpendicular to the surface with the $\mathrm{C}$ end of the molecule closest to the surface. At each point we fix the position of the $\mathrm{C}$ atom and relax the positions of all the $\mathrm{O}$ atoms and the top two Ru layers. The results are shown in the left panel of Fig. 1, where we have also considered the path for $\mathrm{CO}$ directly above an adsorbed $\mathrm{O}$ atom. It can be seen that $\mathrm{CO}$ experiences an energy barrier which starts to build up at about $2.5 \AA$ from the surface for all sites, reflecting a repulsive interaction with the O-covered surface. Furthermore, it is apparent that the surface potential is significantly corrugated: Considering a constant-total-energy surface as a function of the lateral position of the $\mathrm{CO}$, we find that it exhibits the lowest energy (but always repulsive) over the fcc-hollow site. Thus $\mathrm{CO}$ tends to avoid the $\mathrm{O}$ adatoms but will not form a chemical bond with the metal substrate. The

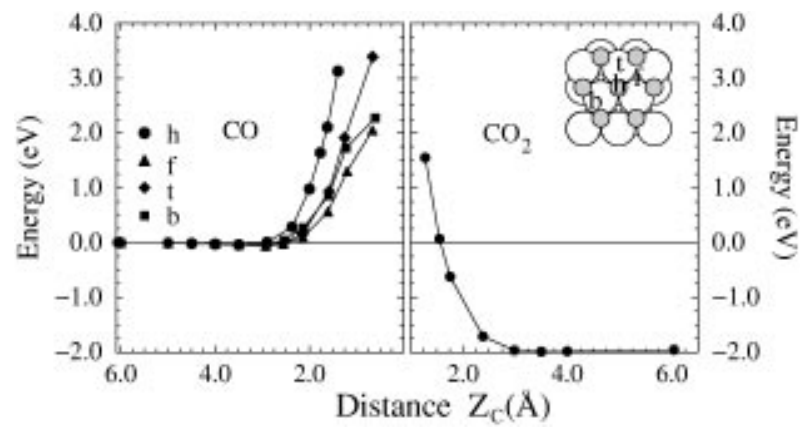

FIG. 1. Energy as a function of distance of the $\mathrm{C}$ atom, $Z_{C}$, of the $\mathrm{CO}$ and $\mathrm{CO}_{2}$ molecules from the surface for the various sites tested. The molecular axes are constrained to be perpendicular to the surface. The zero of energy refers to the situation where $\mathrm{CO}$ is far away from the $(1 \times 1)-\mathrm{O} / \mathrm{Ru}(0001)$ surface $\left(Z_{\mathrm{C}} \approx 6 \AA\right)$.
O-covered surface thus prevents reaction via the $\mathrm{L}-\mathrm{H}$ process leaving the possibility for reaction via gas-phase $\mathrm{CO}$ with chemisorbed O, i.e., the E-R reaction. In this respect, for the approach of $\mathrm{CO}$ directly above an adsorbed $\mathrm{O}$ atom (full circles in Fig. 1), we find the initial, repulsive interaction turns into an attractive one, and the $\mathrm{CO}$ molecule and the adsorbed oxygen atom react to form $\mathrm{CO}_{2}$. That is, the $\mathrm{O}-\mathrm{Ru}$ adsorption bond switches over to form the $\mathrm{CO}_{2}$ complex. (This is discussed in more detail below.) On relaxing the position of the $\mathrm{C}$ atom, the $\mathrm{CO}_{2}$ molecule then leaves the surface with a significant energy gain of $\approx 1.95 \mathrm{eV}$. The associated energetics are shown in the right panel of Fig. 1. It is important here to emphasize that we have considered all relevant reaction paths for $\mathrm{CO}$ at the $(1 \times 1)-\mathrm{O} / \mathrm{Ru}(0001)$ surface and that although on first consideration Fig. 1 may appear to suggest that the favored reaction pathway for $\mathrm{CO}_{2}$ formation is over sites away from the adsorbed $\mathrm{O}$ atom, in particular the fcc-hollow site, this is not the case: Indeed "slow moving" CO molecules with low translational energy will be "steered" towards the fcc-hollow sites. These molecules will, however, not achieve reaction due to the sizable energy barrier. Fast $\mathrm{CO}$ molecules of high translational energy, not susceptible to steering effects, which are incident at sites away from an adsorbed $\mathrm{O}$ atom will also not react, but will rather be reflected from the surface. Thus to produce $\mathrm{CO}_{2}$ via this mechanism, the results indicate that the molecule must "hit," or get very close to, an adsorbed O atom. Interestingly, the calculations show that there is a physisorption well for $\mathrm{CO}$, as well as for $\mathrm{CO}_{2}$, above the surface (barely visible in Fig. 1). The wells are very shallow $(\approx 0.04 \mathrm{eV})$ and thus they will not play a role. It should be noted, however, that the calculated depths are likely to be lower bounds because the employed exchange-correlation functional does not describe the long-range (van der Waals type) interactions and the physisorption wells are found at distances where the true potential energy is likely to be more attractive than that given by the DFT-GGA calculation.

A more detailed understanding of the pathway for reaction via scattering of $\mathrm{CO}$ is obtained by evaluating an appropriate cut through the high-dimensional potential energy surface (PES); thus cut is defined by two variables: the vertical position of the $\mathrm{C}$ atom and the vertical position of the $\mathrm{O}$ adatom below the molecule. In order of ease of analysis, the $\mathrm{CO}$ axis is initially held perpendicular to the surface. The resulting PES is presented in Fig. 2, where the coordinate system is shown as the inset. For each point we relaxed all the $\mathrm{O}$ atoms (except that held fixed at $Z_{O}$ ), and the top two Ru layers. The repulsive interaction is again evident as CO nears the surface. In response to the approaching molecule, the $\mathrm{O}$ adatom moves in towards the surface: For example, at a distance of $Z_{\mathrm{C}}=1.9 \AA$, the $\mathrm{O}$ atom is displaced inwards by $Z_{\mathrm{O}}=0.2 \AA$. Thus the impinging CO molecule "hits" a "soft wall." Reaction to $\mathrm{CO}_{2}$ is achieved via an upward 


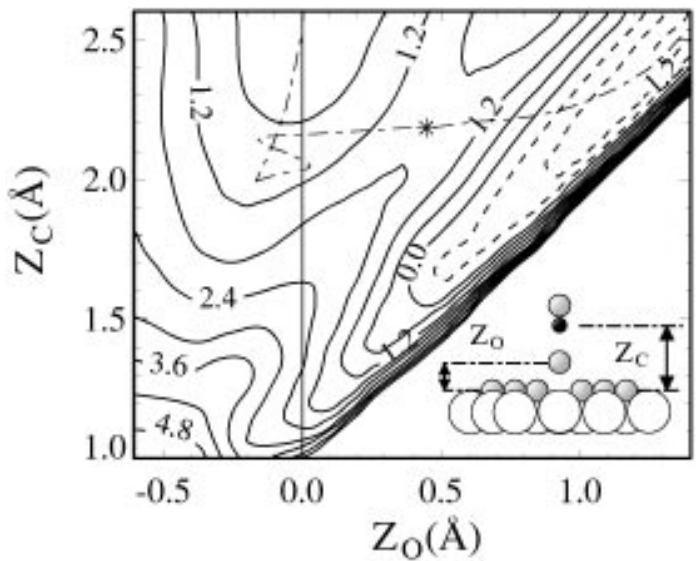

FIG. 2. Cut through the high-dimensional potential energy surface (PES) as a function of the positions of the $\mathrm{C}$ atom, $Z_{C}$, and the $\mathrm{O}$ adatom, $Z_{\mathrm{O}}$ (see inset). The molecular axes are constrained to be perpendicular to the surface. Positive energies are shown as continuous lines, negative ones as dashed lines. The contour-line spacing is $0.6 \mathrm{eV}$. The dot-dashed line indicates a possible reaction pathway.

movement or "hop" of the O adatom by $\approx 0.4 \AA$ towards the $\mathrm{CO}$ molecule (corresponding to movement parallel to the horizontal axis of Fig. 2) and brings the system to the transition state of the reaction marked by the asterisk. In view of the similar masses of $\mathrm{O}$ and $\mathrm{C}$, it is likely that the impinging $\mathrm{CO}$ molecule will impart a significant amount of energy to the $\mathrm{O}$ adatom, thus stimulating its vibrations and facilitating its motion (indicated by the oscillations in the dot-dashed curve). The newly formed $\mathrm{CO}_{2}$ molecule then finds itself in a particularly unfavorable position and is strongly repelled from the surface towards the vacuum region with a large energy gain of $1.95 \mathrm{eV}$. In the cut through the PES shown in Fig. 2, the energy barrier hindering $\mathrm{CO}_{2}$ formation is $\approx 1.6 \mathrm{eV}$.

The PES of Fig. 2 corresponds to a constrained situation of the surface-CO angle. When this constraint is dropped, i.e., when the tilt angle of the $\mathrm{CO}$ axis is allowed to relax [18], we find that the energy barrier is reduced to $1.1 \mathrm{eV}$, and also that the position of the saddle point of the PES occurs closer to the surface (by $0.3 \AA$ ). At the transition state (see Fig. 3), the optimum tilt angle with respect to the surface normal is found to be $49^{\circ}$, which corresponds to a bond angle of $131^{\circ}$ for the " $\mathrm{CO}_{2}$-like" complex. Interestingly, this geometry is very similar to that associated with the $\mathrm{CO}_{2}{ }^{-}$ion [19] and to that proposed for the "activated complex" for the $\mathrm{CO}$ oxidation reaction over other transition metal surfaces [20].

We have thus identified a likely reaction pathway for the E-R mechanism. The activation energy barrier for this type of reaction appears to be sizable. However, it is very similar to those derived from experimental studies of $\mathrm{CO}$ oxidation reactions at other surfaces [3] which proceed via a L-H process, and also for the measurements at $\mathrm{Ru}(0001)$ the estimated activation energy is comparable, namely $0.85 \mathrm{eV}[3,5,6]$. On the basis of the present results we

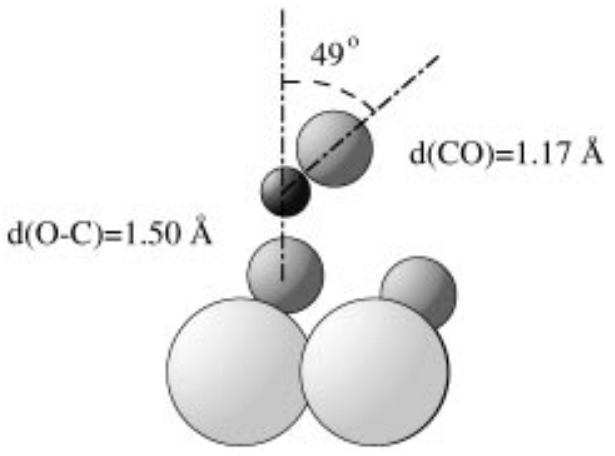

FIG. 3. Transition state geometry identified for the reaction of gas-phase $\mathrm{CO}$ with adsorbed oxygen when the constraint on the molecular axis is relaxed. The large, medium, and small circles represent $\mathrm{Ru}, \mathrm{O}$, and $\mathrm{C}$ atoms, respectively.

predict an energy diagram for the E-R mechanism, which is shown in Fig. 4. An estimate of the reaction rate gives $R=7.5 \times 10^{6} \exp \left[-1.1 /\left(k_{B} T\right)\right] \mathrm{s}^{-1}$ which yields at $T=500 \mathrm{~K}, R=6 \times 10^{-5} \mathrm{CO}_{2}$ molecules formed per surface $\mathrm{Ru}$ atom per second [21] which is about $3 \times 10^{-6}$ smaller than that observed experimentally [5]. This indicates that this mechanism alone cannot explain the particularly high $\mathrm{CO}_{2}$ turnover rate. Nevertheless, the rate is only about a factor of $10^{-3}$ less than that for the $\mathrm{L}-\mathrm{H}$ process at $\mathrm{Pt}$ or $\mathrm{Pd}$ [3] and with molecular beam experiments this predicted E-R mechanism and associated energetics could possibly be measured for the first time for the $\mathrm{CO}$ oxidation reaction.

To understand the high reaction rate reported experimentally, we turn to another consideration: $\mathrm{CO}$ molecules might adsorb at sites at which an oxygen atom has been removed (e.g., by the above described E- $\mathrm{R}$ reaction). Indeed, assuming thermal equilibrium of the $\mathrm{CO}+\mathrm{O}_{2}$ gas and a mixed $\mathrm{CO}+\mathrm{O}$ adlayer, the law of mass action indicates that about $0.03 \%$ of the sites of the $(1 \times 1)$ adlayer will be occupied by $\mathrm{CO}$ (we assumed that the $\mathrm{O}_{2}$ and $\mathrm{CO}$ partial pressures are equal, the temperature is $T=500 \mathrm{~K}$, and the binding energy of $\mathrm{CO}$ into an $\mathrm{O}$ vacancy of the $(1 \times 1)$ adlayer is calculated to be $0.85 \mathrm{eV}$ and the adsorption energy of $\frac{1}{2} \mathrm{O}_{2}$ (i.e., an $\mathrm{O}$ atom) into a vacancy is $1.20 \mathrm{eV}$. In reality the $\mathrm{CO}$ concentration will

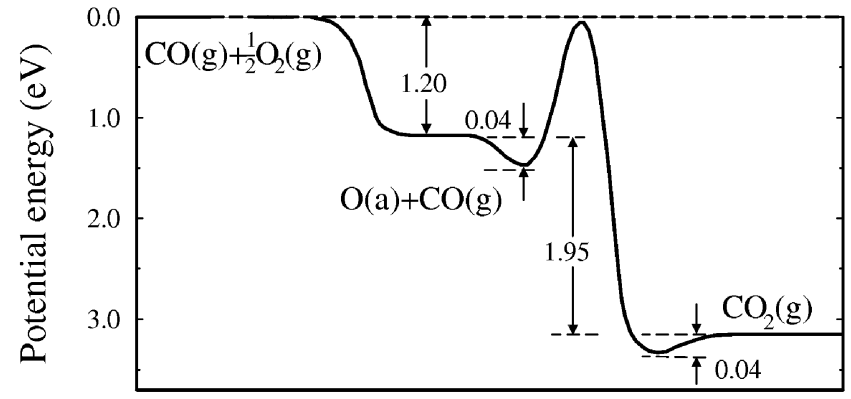

Reaction coordinate

FIG. 4. Calculated energy diagram for the E-R mechanism of $\mathrm{CO}$ oxidation at $\mathrm{Ru}(0001)$. Note that the depths of the physisorption wells are exaggerated for clarity. 
be even higher because catalysis is not ruled by thermodynamic equilibrium but by kinetics, and we find that $\mathrm{CO}$ adsorption into an existing $\mathrm{O}$ vacancy can proceed basically without hindrance while $\mathrm{O}$ adsorption (from $\mathrm{O}_{2}$ ) is hindered by an energy barrier (see, e.g., Ref. [16]). Therefore the actual percentage of surface sites occupied by $\mathrm{CO}$ will be somewhat larger. For these $\mathrm{CO}$ molecules there is a substantial attempt frequency to form a $\mathrm{CO}_{2}$ molecule with neighboring $\mathrm{O}$ adatoms, now by the $\mathrm{L}-\mathrm{H}$ mechanism which we expect to proceed very efficiently due to the relatively weak binding energy of both $\mathrm{CO}$ (which we calculate to be about half that which it has on the clean surface - and on the surface with $O$ coverages: $\Theta \leq 0.5$ ) and $\mathrm{O}$ atoms in the high-coverage $(1 \times 1)$ adlayer, as well as the close proximity of the constituents. We find that the energy gain on $\mathrm{CO}_{2}$ formation (of the surface reaction) via this mechanism is about $0.66 \mathrm{eV}$ [22]; noticeably smaller than that of $1.95 \mathrm{eV}$ (see Fig. 4) but still quite significant if compared to that of $\approx 0.2 \mathrm{eV}$ at $\mathrm{Pt}(111)$ and $\mathrm{Pd}(111)$ [1] as determined experimentally.

In summary, we now have the following picture of $\mathrm{CO}$ oxidation at $\mathrm{Ru}(0001)$ : With respect to other transition metals, ruthenium binds oxygen particularly strongly. Therefore, at low oxygen coverages a $\mathrm{Ru}$ catalyst dissociates $\mathrm{O}_{2}$ efficiently, but (in contrast to, e.g., Pd) it holds the oxygen (and $\mathrm{CO}$ ) so strongly that reaction to $\mathrm{CO}_{2}$ is disfavored. A good catalyst should actuate this dissociation but at the same time should not bind the dissociated entities too strongly, which gives them good capability to diffuse and react. Too strongly bound constituents would have little reason to react at all. For oxygen in the $(1 \times 1)$-O monolayer, the adsorption energy is significantly weaker and thus $\mathrm{CO}_{2}$ formation enhanced. Our results indicate that this high coverage oxygen phase enables reaction via both scattering of $\mathrm{CO}$ (the E-R mechanism) and by the L-H mechanism where the former may play an important role in initiating the reaction. The high rate then develops and is maintained by an efficient L-H mechanism. Our theoretical results thus explain the anomalous dependence of the reaction on oxygen pressure, as only under sufficiently high oxygen pressure the $(1 \times 1)$ layer is attained.

The different mechanisms identified in our study are also likely to play important roles for other catalytic reactions. We hope that the detailed predictions and the unusual mechanism outlined above will be tested by additional experiments.

We wish to thank Martin Fuchs for his help in creating the pseudopotentials.

[1] T. Engel and G. Ertl, Adv. Catal. 28, 1 (1979); The Chemical Physics of Solid Surfaces and Heterogeneous Cataly- sis, edited by D. A. King and D. P. Woodruff (Elsevier, New York, 1982), Vol. 4, and references therein.

[2] A. Bielánski and J. Haber Oxygen in Catalysis, edited by H. Heinemann (Marcel Dekker Inc., New York, 1991), Chap. 6.

[3] C. H.F. Peden, in Surface Science of Catalysis: In Situ Probes and Reaction Kinetics, edited by D. J. Dwyer and F. M. Hoffman (American Chemical Society, Washington, DC, 1992).

[4] See, e.g., Frontiers in Catalysis: Ammonia Synthesis and Beyond, edited by $\mathrm{H}$. Topsoe, M. Boudart, and J. K. Nørskov, Topics in Catalysis Vol. 1 (Baltzer, Basel, Switzerland, 1994), p. 185.

[5] C. H. F. Peden and D. W. Goodman, J. Phys. Chem. 90, 1360 (1986).

[6] C. H.F. Peden, D. W. Goodman, M. D. Weisel, and F. M. Hoffman, Surf. Sci. 253, 44 (1991).

[7] E. W. Kuipers, A. Vardi, A. Danon, and A. Amirav, Phys. Rev. Lett. 66, 116 (1991); C. T. Rettner, Phys. Rev. Lett. 69, 383 (1992); C. T. Rettner and D. J. Auerbach, Phys. Rev. Lett. 74, 4551 (1995); C. T. Rettner, J. Chem. Phys. 101, 1529 (1994).

[8] J. P. Perdew, J. A. Chevary, S. H. Vosko, K. A. Jackson, M. R. Pederson, D. J. Singh, and C. Fiolhais, Phys. Rev. B 46, 6671 (1992).

[9] N. Troullier and J.L. Martins, Phys. Rev. B 43, 1993 (1993).

[10] S. L. Cunningham, Phys. Rev. B 10, 4988 (1974).

[11] C. Stampfl and M. Scheffler, Phys. Rev. B 54, 2868 (1996).

[12] J. Neugebauer and M. Scheffler, Phys. Rev. B 46, 16067 (1992).

[13] R. Stumpf and M. Scheffler, Comput. Phys. Commun. 79, 447 (1994).

[14] C. Stampfl and M. Scheffler (to be published).

[15] H. Pfnür, G. Held, M. Lindroos, and D. Menzel, Surf. Sci. 220, 43 (1989).

[16] C. Stampfl, S. Schwegmann, H. Over, M. Scheffler, and G. Ertl, Phys. Rev. Lett. 77, 3371 (1996).

[17] C. Stampfl, Surf. Rev. Lett. 3, 1567 (1996).

[18] Tilts of the CO axis were optimized in two planes, with essentially the same result. The planes were those passing through the fcc-hollow and the on-top sites. The lateral position of the $\mathrm{C}$ atom was fixed to be the same as that of the $\mathrm{O}$ adatom.

[19] J. Pacansky, U. Wahlgren, and P. S. Bagus, J. Chem. Phys. 62, 2740 (1975).

[20] G. W. Coulston and G. L. Haller, J. Chem. Phys. 95, 6932 (1991), and references therein.

[21] For our estimate we use the frequency of impinging $\mathrm{CO}$ molecules per surface $\mathrm{Ru}$ atom of $\nu=7.5 \times 10^{6} \mathrm{~s}^{-1}$ which corresponds to a $\mathrm{CO}$ gas phase pressure of 16 torr.

[22] This value is obtained as the reaction energy of $\mathrm{CO}+$ $\frac{1}{2} \mathrm{O}_{2} \rightarrow \mathrm{CO}_{2}$ in vacuum, minus the energy gained on adsorption of $\frac{1}{2} \mathrm{O}_{2}$ and $\mathrm{CO}$ onto the surface. 